\title{
Uso e dependência do smartphone de adolescentes no cenário escolar de tempo
}

\section{integral}

\author{
Smartphone use and dependence among adolescents attending full-time school setting \\ Uso de teléfonos inteligentes y dependencia de los adolescentes en el entorno escolar a tiempo \\ completo
}

Recebido: 14/05/2021 | Revisado: 25/05/2021 | Aceito: 28/05/2021 | Publicado: 11/06/2021

Paula Pessoa de Brito Nunes

ORCID: https://orcid.org/0000-0002-5189-8469

Centro Universitário Ateneu, Brasil

E-mail: paulanunes2912@gmail.com

Ana Paula Vasconcellos Abdon

ORCID: https://orcid.org/0000-0002-1597-1817

Universidade de Fortaleza, Brasil

E-mail: paulaabdon@unifor.br

Francisco Valter Miranda Silva

ORCID: https://orcid.org/0000-0002-7122-2466 Universidade de Fortaleza, Brasil

E-mail: valtermiranda15@gmail.com

Daniele de Queiroz Martins

ORCID: https://orcid.org/0000-0001-5732-4581

Centro Universitário Ateneu, Brasil E-mail: daniqfisio@gmail.com

Claudiana Batista de Brito

ORCID: https://orcid.org/0000-0002-1827-8276

Centro Universitário Ateneu, Brasil E-mail: cbbdiana@gmail.com

Mirna Albuquerque Frota

ORCID: https://orcid.org/0000-0003-3004-2554

Universidade de Fortaleza, Brasil

E-mail: mirnafrota@unifor.br

\begin{abstract}
Resumo
Objetivos: Conhecer o perfil de uso e a dependência do smartphone de adolescentes em escolas de tempo integral. Método: Estudo transversal realizado em seis Escolas Estaduais de Educação Profissional (EEEP) na cidade de Fortaleza, Ceará, Brasil, desenvolvido entre setembro e outubro de 2019. Participaram 286 adolescentes (15-19 anos) que responderam dois questionários (dados sociodemográficos, uso e dependência do smartphone). Utilizou-se o SPSS 23.0 para análise descritivas e inferenciais, tendo como desfecho a dependência do smartphone. Resultados: Houve maior percentual do sexo masculino $(53,5 \%)$, idade de 16 anos $(31,5 \%)$ e classe social D $(52,4 \%)$. Sobre a utilização, 96,2\% usavam para acessar redes sociais, 71,0\% para receber e fazer ligação, 68,9\% para jogar e 66,1\% para assistir filmes. Verificou-se que $62,6 \%$ apresentavam dependência do smartphone, com tempo de uso de 7,3 ( \pm $3,4)$ horas/diárias. Foi constatada associação entre a dependência do smartphone com a idade (OR: 1,48; $\mathrm{p}=0,009$ ), utilizar fones de ouvido (OR: 2,76; $\mathrm{p}<0,001)$, e usar o smartphone $1 \mathrm{~h} 18$ minutos a mais $(\mathrm{p}=0,003)$. Conclusão: Dentre as razões para o uso do smartphone pelos adolescentes destacam-se acessar as redes sociais, assistir filmes e jogar, bem como usar WhatsApp para fins de comunicação. Evidenciou-se elevada dependência e tempo de uso do smartphone nos adolescentes, associado a idade e ao uso de fones de ouvido. Assim, alerta-se para a necessidade de campanhas educativas e orientações sobre o uso consciente do smartphone visando a prevenção e redução de agravos à saúde física e mental.
\end{abstract}

Palavras-chave: Smartphone; Medicina da dependência; Adolescente.

\begin{abstract}
Objectives: To know the profile of smartphone use and dependence of adolescents in full-time schools. Method: Cross-sectional study carried out in six State Schools of Professional Education (EEEP) in the city of Fortaleza, Ceará, Brazil, developed between September and October 2019. 286 adolescents (15-19 years old) participated who answered two questionnaires (sociodemographic data, use and smartphone dependency). SPSS 23.0 was used for descriptive and inferential analysis, with the outcome being smartphone dependence. Results: There was a higher percentage of males $(53.5 \%)$, age of 16 years $(31.5 \%)$ and social class D (52.4\%). Regarding usage, $96.2 \%$ used to
\end{abstract}


access social networks, $71.0 \%$ to receive and make calls, $68.9 \%$ to play games and $66.1 \%$ to watch movies. It was found that $62.6 \%$ had smartphone dependence, with $7.3( \pm 3.4)$ hours / daily use time. An association was found between smartphone dependence and age (OR: 1.48; $\mathrm{p}=0.009)$, using headphones (OR: $2.76 ; \mathrm{p}<0.001)$, and using the smartphone $1 \mathrm{~h} 18$ minutes more $(\mathrm{p}=0.003)$. Conclusion: Among the reasons for the use of smartphones by teenagers are accessing social networks, watching movies and playing games, as well as using WhatsApp for communication purposes. High dependence and time of smartphone use in adolescents was evidenced, associated with age and the use of headphones. Thus, it warns of the need for educational campaigns and guidance on the conscious use of the smartphone aiming at the prevention and reduction of physical and mental health problems.

Keywords: Smartphone; Addiction medicine; Adolescent.

\section{Resumen}

Objetivos: Conocer el perfil de uso y dependencia de teléfonos inteligentes entre adolescentes en escuelas de tiempo completo. Método: Estudio transversal realizado en seis Escuelas Públicas de Educación Profesional (EEEP) de la ciudad de Fortaleza, Ceará, Brasil, desarrollado entre septiembre y octubre de 2019. Participaron 286 adolescentes (15-19 años) que respondieron dos cuestionarios (datos sociodemográficos, uso y dependencia de teléfonos inteligentes). Se utilizó SPSS 23.0 para el análisis descriptivo e inferencial, y el resultado fue la dependencia de teléfonos inteligentes. Resultados: Hubo mayor porcentaje de varones $(53,5 \%)$, edad de 16 años $(31,5 \%)$ y clase social D (52,4\%). En cuanto al uso, el 96,2\% solía acceder a las redes sociales, el 71,0\% para recibir y hacer llamadas, el $68,9 \%$ para jugar y el $66,1 \%$ para ver películas. Se encontró que el 62,6\% tenía dependencia del smartphone, con 7,3 $( \pm 3,4)$ horas / tiempo de uso diario. Se encontró asociación entre la dependencia del teléfono inteligente y la edad (OR: 1,48; $\mathrm{p}=0,009)$, el uso de auriculares (OR: 2,76; $\mathrm{p}<0,001)$ y el uso del smartphone $1 \mathrm{~h} 18$ minutos más $(\mathrm{p}=0,003)$. Conclusión: Entre las razones para el uso de teléfonos inteligentes por parte de los adolescentes se encuentran el acceso a las redes sociales, ver películas y jugar juegos, así como utilizar WhatsApp con fines de comunicación. Se evidenció alta dependencia y tiempo de uso de teléfonos inteligentes en adolescentes, asociada a la edad y al uso de auriculares. Así, advierte de la necesidad de campañas educativas y de orientación sobre el uso consciente del smartphone para prevenir y reducir los problemas de salud física y mental.

Palabras clave: Smartphone; Medicina de la dependencia; Adolescente.

\section{Introdução}

O uso das tecnologias tornou-se indispensável na vida humana, os dispositivos eletrônicos têm se modernizado a cada dia, e trazem soluções que envolvem comodismo e praticidade a um toque de tela (Cha \& Seo, 2018). Destaca-se entre essas tecnologias o smartphone, que compacta em um único dispositivo uma variedade de aplicativos que vinculados a Internet, possibilitando a realização de atividades como estudo, trabalho, lazer, dentre outras (Gutiérrez, de Fonseca, \& Rubio, 2016).

O número de usuários de smartphone no mundo ultrapassou os cinco bilhões em 2021, estimando aumento deste número nos anos seguintes (We Are Social, 2021). Este é o principal meio de acesso à rede, sendo utilizado por diferentes faixas etárias (Xie, Szeto, \& Dai, 2017). No Brasil, no ano de 2017 o uso domiciliar da Internet pelos brasileiros era de 74,9\%, havendo um aumento significativo no ano seguinte para 79,1\% (Instituto Brasileiro e Geografia Estatística, 2018).

Dentre os usuários, o adolescente é fortemente atraído para o uso do smartphone, alinhado ao vício da Internet que ocorre em escala global, sendo os aplicativos de jogos e de redes sociais como Facebook, Instagram entre outros, os que mais chamam a atenção desta população (Comitê Gestor da Internet, 2019). Além destes, o WhatsApp se tornou uma ferramenta de comunicação muito utilizada, seja para fins de relacionamentos pessoais ou trabalho (Lee, Kim, \& Choi, 2017). Outra particularidade inerente aos adolescentes, é que este dispositivo é comumente utilizado para assistir filmes, leituras e estudos (Jiang \& Zhao, 2016).

A interação do adolescente com o smartphone ocorre por diferentes formas e finalidades, tornando-os potencialmente dependentes e por tempo excessivo (Elhai, Dvorak, Levine, \& Hall, 2017a). Tal comportamento pode gerar agravos à saúde, dentre eles a queixa de dor cervical, má qualidade do sono, prejuízo no rendimento escolar e problemas de relacionamentos (Derevensky, Hayman, \& Lynette Gilbeau, 2019; Schweizer, Berchtold, Barrense-Dias, Akre, \& Suris, 2017).

Diante desse cenário, em consonância com os alertas e recomendação da Organização Mundial de Saúde (OMS) (WHO, 2014) e da Sociedade Brasileira de Pediatria (Sociedade Brasileira de Pediatria, 2016) no que concerne ao uso de dispositivos eletrônicos, dentre eles o smartphone, este estudo teve como objetivo conhecer o perfil de uso e a dependência do 
smartphone de adolescentes de escola de tempo integral.

\section{Metodologia}

Realizou-se um estudo quantitativo do tipo transversal, oriundo de um projeto de pesquisa intitulado "Estudo das alterações posturais e álgicas na região cervical associada ao uso de smartphone em adolescentes", desenvolvido em Escolas Estaduais de Educação Profissional (EEEPs) de ensino médio da cidade de Fortaleza, Ceará, Brasil. Este desenho foi escolhido por propiciar análises de correlações de variáveis por meio de técnicas estatística que procuram explicar a relação existente entre elas, os efeitos e testar hipóteses (Bloch \& Klein, 2009). O recrutamento dos participantes e coleta de dados foram realizados nos meses de agosto e setembro de 2019.

A partir de 2008, as EEEPs foram instituídas no Ceará visando integrar o ensino médio à educação profissional em tempo integral. No Ceará, são 115 EEEPs existentes em todo o estado, seu funcionamento é em tempo integral, integrando o ensino médio à educação profissional proporcionando um futuro mais justo e com mais oportunidades aos jovens cearenses. Atualmente na cidade de Fortaleza existem 21 EEEPs, distribuídas nas 6 secretarias executivas regionais (Secretaria de Educação do Estado do Ceará, 2019).

Para o estudo, a seleção dos adolescentes foi feita por conglomerado em dois estágios. No primeiro estágio, foram selecionadas seis escolas por sorteio, sendo uma em cada regional, com exceção da regional Centro que não possui. No segundo, foi selecionada uma turma por série $\left(1^{\mathrm{a}}\right.$ a $\left.3^{\mathrm{a}}\right)$, manhã e tarde, totalizando três turmas por EEEPs. O recrutamento dos participantes iniciou-se por chamamento público para uma palestra explicativa dirigida aos pais/responsáveis e adolescentes, seguido das solicitações de consentimento de ambos.

Participaram do estudo 286 adolescentes ( 15 a 19 anos), frequentando da $1^{\mathrm{a}}$ a $3^{\mathrm{a}}$ série do ensino médio, matriculados no ano letivo de 2019 nas EEEPs selecionadas e que possuíssem smartphone, compondo uma amostra probabilística, distribuídos equitativamente entre as séries ( 96 da $1^{\mathrm{a}}$ série, 95 da $2^{\mathrm{a}}$ série e 95 da $3^{\mathrm{a}}$ série do ensino médio). Os critérios de exclusão estabelecidos foram o não comparecimento a escola nos dias de coleta; autorrelato de doenças prévias (escoliose, fratura/lesões degenerativas na coluna cervical e lesões traumáticas recentes). Além disso, gestantes, portadores de deficiência física, visual, auditiva e cognitiva não participaram do estudo devido à falta de adaptabilidade dos instrumentos de coleta estabelecidos no projeto guarda-chuva.

O quantitativo de adolescentes foi estimado por cálculo amostral, considerando uma população finita ( $\mathrm{n}=224.153)$ de adolescentes (15 a 19 anos) da cidade de Fortaleza, prevalência de 18\% de dor cervical em usuários de dispositivos móveis (Xie et al., 2017), precisão amostral de 5\%, intervalo de confiança de $95 \%$ e acréscimo de $10 \%$ para perda amostral, sendo necessário um mínimo de 249 adolescentes.

Os participantes responderam dois instrumentos de coleta de dados autoaplicáveis, na própria sala de aula da escola, em horário determinado pela direção a fim de garantir a não interferência nas atividades escolares. Os instrumentos de coleta foram o questionário sociodemográfico (sexo, idade, raça, classe social, tipo de internet, perfil e tempo de uso em horas do smartphone) e o Smartphone Addiction Inventory (SPAI-BR).

O Smartphone Addiction Inventory (SPAI-BR) é um questionário de domínio público, validado e adaptado para o português em uma população jovem, que possui um questionário com 26 itens com resposta sim (1)/não (0) que objetiva avaliar a dependência do smartphone, dividido em quatro subescalas que mensuram os constructos comportamento compulsivo (itens $5,6,7,10,11,18,20,21$ e 22), comprometimento funcional (itens 8, 12, 13, 15, 17, 23, 24 e 26), síndrome de abstinência (itens 2, 4, 14, 16, 19, e 25) e síndrome de tolerância (itens 1, 3 e 9). A pontuação final foi o somatório dos itens, e o ponto de corte adotado para a dependência do smartphone foi de sete pontos, que possui sensibilidade de $90,54 \%$ e especificidade de 59,93\%. Este questionário foi selecionado também por ser o único adaptado e validado para o português 
durante o período de aprovação ética e coleta dos dados (Khoury et al., 2017).

Os dados foram analisados pela estatística descritiva e inferencial usando o SPSS Statistics IBM ${ }^{\circledR}$ versão 23.0. Na análise univariada, foram calculados os valores absolutos (n) e relativos (\%) das variáveis categóricas, e as médias \pm desvio padrão (DP) das variáveis numéricas. Na análise bivariada, foram calculadas as medidas de associação odds ratio (OR) e os seus respectivos intervalos de confiança de 95\% (IC95\%) por meio do teste qui-quadrado para verificar a associação entre o desfecho dependência do smartphone e as variáveis sociodemográficas e o perfil de uso. Para analisar a relação entre o desfecho com o tempo de uso do smartphone aplicou-se o teste t, após teste de normalidade de Kolmogorov-Smirnov. Adotouse significância de $5 \%(p<0,05)$.

O estudo foi aprovado pelo Comitê de Ética em Pesquisas em Seres Humanos da Universidade de Fortaleza COÉTICA/UNIFOR com parecer nº. 3.341 .394 em consonância com a Resolução 466/12 do Conselho Nacional de Saúde. Pais/responsáveis e adolescentes consentiram sua participação pela assinatura dos termos de consentimento Livre e Esclarecido e Termo de Assentimento, respectivamente.

\section{Resultados}

Referente às variáveis sociodemográficas, houve maior percentual do sexo masculino ( $\mathrm{n}=153 ; 53,5 \%)$, idade de 16 anos $(\mathrm{n}=90 ; 31,5 \%)$, pertencentes a classe social $\mathrm{D}(\mathrm{n}=150 ; 52,4 \%)$, de cor autorreferida parda $(\mathrm{n}=154 ; 53,8 \%)$. Do total, $96,5 \%$ ( $n=276)$ possuíam Internet em casa e 48,6\% (n=139) do tipo móvel (Tabela 1).

Tabela 1. Distribuição das variáveis sociodemográficas dos adolescentes de escolas de tempo integral. Fortaleza, Ceará, 2019.

\begin{tabular}{lcc}
\hline Variáveis & $\mathbf{n}$ & $\mathbf{\%}$ \\
\hline Sociodemográficas & & \\
Sexo \\
masculino & 153 & 53,5 \\
feminino & 133 & 46,5 \\
Idade & & 28,0 \\
15 & 80 & 31,5 \\
16 & 90 & 30,8 \\
17 & 88 & 7,7 \\
18 & 22 & 2,1 \\
19 & 6 & 53,8 \\
Raça autorreferida & 154 & 25,2 \\
parda & 72 & 13,6 \\
branca & 39 & 4,2 \\
preta & 12 & 3,1 \\
amarela & 9 & 3,1 \\
indígena & & 96,9 \\
Classe social & 9 & 96,5 \\
A/B & 277 & 3,5 \\
C/D/E & & \\
Possui Internet em casa & 276 & 48,6 \\
sim & 10 & 51,4 \\
não & & \\
Possui Internet móvel & 139 & \\
sim & 147 & \\
não & & \\
\hline
\end{tabular}

Fonte: Autores.

Em relação ao perfil de uso do smartphone, as principais razões para a utilização foram acessar redes sociais ( $\mathrm{n}=275$; 96,2\%), fazer e receber ligação ( $n=203 ; 71,0 \%)$, assistir filmes $(n=197 ; 68,9 \%)$ e jogar $(n=189 ; 66,1 \%)$. Como forma de 
comunicação utilizavam o WhatsApp $(n=105 ; 36,7 \%)$. Em relação à dependência do smartphone, verificou-se uma prevalência de $62,6 \%(n=179)$, sendo constatado que os adolescentes passavam em média 7,3 $( \pm 3,4)$ horas/diárias usando o dispositivo (Tabela 2).

Tabela 2. Distribuição do perfil e tempo de uso do smartphone dos adolescentes de escolas de tempo integral. Fortaleza, Ceará, 2019.

\begin{tabular}{|c|c|c|c|}
\hline Variáveis & $\mathbf{n}$ & $\%$ & Média \pm DP \\
\hline Idade do primeiro smartphone & & & $11,2 \pm 2,07$ \\
\hline \multicolumn{4}{|l|}{ Razões para o uso } \\
\hline fazer e receber ligações & 203 & 71,0 & \\
\hline jogar & 189 & 66,1 & \\
\hline assistir filmes & 197 & 68,9 & \\
\hline acessar redes sociais & 275 & 96,2 & \\
\hline utilizar fone de ouvido & 208 & 72,7 & \\
\hline deixar o celular ligado à noite & 215 & 75,2 & \\
\hline \multicolumn{4}{|l|}{ Forma de comunicação } \\
\hline ligação & 4 & 1,4 & \\
\hline WhatsApp & 105 & 36,7 & \\
\hline redes sociais & 26 & 9,1 & \\
\hline outros & 1 & 0,3 & \\
\hline duas ou mais & 150 & 52,4 & \\
\hline \multicolumn{4}{|l|}{ Dependência do smartphone } \\
\hline não & 179 & 62,6 & \\
\hline $\operatorname{sim}$ & 107 & 37,4 & \\
\hline Tempo de uso diário & & & $7,3 \pm 3,4$ \\
\hline
\end{tabular}

DP: desvio padrão. Fonte: Autores.

No tocante às subescalas do SPAI-BR, 37,4\% $(\mathrm{n}=107)$ dos adolescentes apresentaram indicação de comportamento compulsivo segundo o percentual de resposta ao item "usar o smartphone exerceu efeitos negativos nos meus relacionamentos interpessoais"; mais de 30\% tiveram indicação de comprometimento funcional a partir do percentual das respostas aos itens "uso de smartphone exerceu certos efeitos negativos no desempenho escolar ou no trabalho" $(30,1 \% ; \mathrm{n}=86)$ e "interação com os membros da família diminuiu devido ao uso de smartphone" $(33,6 \% ; n=96) ; 48,6 \%(n=139)$ mostraram síndrome de abstinência segundo as respostas ao item "ideia de usar smartphone surge como o primeiro pensamento na mente ao acordar"; e 76,9\% (n=220) apresentaram síndrome de tolerância de acordo com as respostas ao item "Já me disseram mais de uma vez que eu passo tempo demais no smartphone" (Tabela 3).

Ademais, houve maior proporção nos itens "eu me sinto disposto a usá-lo mesmo quando me sinto cansado" (60,5\%; $\mathrm{n}=173)$, "sinto dores ou incômodos nas costas ou desconforto nos olhos" $(60,1 \% ; n=172)$ e "mais de uma ocasião eu dormi menos que quatro horas porque fiquei usando o smartphone" $(58,0 \%$; $\mathrm{n}=166)$ (Tabela 3$)$. 
Tabela 3. Distribuição das subescalas e respostas do questionário Smartphone Addiction Inventory (SPAI-BR) dos adolescentes de escolas de tempo integral. Fortaleza, Ceará, 2019.

\begin{tabular}{|c|c|c|}
\hline \multirow{2}{*}{ Subescalas/perguntas } & \multicolumn{2}{|c|}{ Sim } \\
\hline & $\mathbf{n}$ & $\%$ \\
\hline \multicolumn{3}{|l|}{ Comportamento Compulsivo } \\
\hline Eu me sinto disposto a usar o smartphone mesmo quando me sinto cansado & 173 & 60,5 \\
\hline Eu uso smartphone durante mais tempo e/ou gasto mais dinheiro nele do que eu pretendia inicialmente & 55 & 19,2 \\
\hline $\begin{array}{l}\text { Embora o uso de smartphone tenha trazido efeitos negativos nos meus relacionamentos interpessoais, a } \\
\text { quantidade de tempo que eu gasto nele se mantém a mesma }\end{array}$ & 107 & 37,4 \\
\hline Eu me sinto incomodado ou para baixo quando eu paro de usar o smartphone por um certo período de tempo & 74 & 25,9 \\
\hline Eu não consigo controlar o impulso de utilizar o smartphone & 102 & 35,7 \\
\hline Minhas atividades de lazer diminuíram por causa do uso do smartphone & 61 & 21,3 \\
\hline Minha vida seria sem graça se eu não tivesse o smartphone & 133 & 46,5 \\
\hline Navegar no smartphone tem causado prejuízos para a minha saúde física & 39 & 13,6 \\
\hline Eu tenho tentado passar menos tempo usando o smartphone, mas não tenho conseguido & 98 & 34,3 \\
\hline \multicolumn{3}{|l|}{ Comprometimento Funcional } \\
\hline Em mais de uma ocasião, eu dormi menos que quatro horas porque fiquei usando o smartphone & 166 & 58,0 \\
\hline Eu me sinto mais satisfeito utilizando o smartphone do que passando tempo com meus amigos & 33 & 11,5 \\
\hline Eu sinto dores ou incômodos nas costas, ou desconforto nos olhos, devido ao uso excessivo do smartphone & 172 & 60,1 \\
\hline O uso de smartphone tem causado efeitos negativos no meu desempenho na escola ou no trabalho & 86 & 30,1 \\
\hline Minha interação com meus familiares diminuiu por causa do meu uso do smartphone & 96 & 33,6 \\
\hline Eu tornei o uso do smartphone um hábito e minha qualidade e tempo total de sono diminuíram & 105 & 36,7 \\
\hline Eu preciso gastar cada vez mais tempo no smartphone para alcançar a mesma satisfação de antes & 21 & 7,3 \\
\hline Eu me sinto cansado durante o dia devido ao uso do smartphone tarde da noite/de madrugada & 78 & 27,3 \\
\hline \multicolumn{3}{|l|}{ Síndrome de Abstinência } \\
\hline $\begin{array}{l}\text { Eu me sinto desconfortável/ansioso/inquieto quando eu fico sem usar o smartphone durante um certo } \\
\text { período }\end{array}$ & 129 & 45,1 \\
\hline Eu me sinto inquieto e irritado quando não tenho acesso ao smartphone & 106 & 37,1 \\
\hline A ideia de utilizar o smartphone vem como primeiro pensamento na minha cabeça quando acordo de manhã & 139 & 48,6 \\
\hline $\begin{array}{l}\text { Eu me sinto ansioso ou irritável quando meu smartphone não está disponível e sinto falta de algo ao parar o } \\
\text { uso do smartphone por certo período }\end{array}$ & 126 & 44,1 \\
\hline Eu sinto uma grande vontade de usar o smartphone novamente logo depois que eu paro de usá-lo & 116 & 40,6 \\
\hline Eu não consigo fazer uma refeição sem utilizar o smartphone & 72 & 25,2 \\
\hline \multicolumn{3}{|l|}{ Síndrome de Tolerância } \\
\hline Já me disseram mais de uma vez que eu passo tempo demais no smartphone & 220 & 76,9 \\
\hline Eu acho que eu tenho ficado cada vez mais tempo conectado ao smartphone & 164 & 57,3 \\
\hline Eu tenho aumentado consideravelmente o tempo gasto usando o smartphone nos últimos 3 meses & 105 & 36,7 \\
\hline
\end{tabular}

Fonte: Autores.

$\mathrm{Na}$ análise bivariada, verificou-se associação significativa entre a dependência do smartphone com a idade (OR: 1,48; $\mathrm{p}=0,009)$ e utilizar fones de ouvido (OR: 2,76; $<<0,001)$. Além disso, existiu diferença significativa em relação ao tempo de uso, sendo que os adolescentes com dependência do smartphone passavam $1 \mathrm{~h} 18$ minutos a mais do que aqueles com ausência de dependência ( $\mathrm{p}=0,003)$ (Tabela 4$)$. 
Tabela 4. Análise bivariada entre a dependência do smartphone e as variáveis sociodemográficas e perfil de uso do dispositivo dos adolescentes de escolas de tempo integral. Fortaleza, Ceará, 2019.

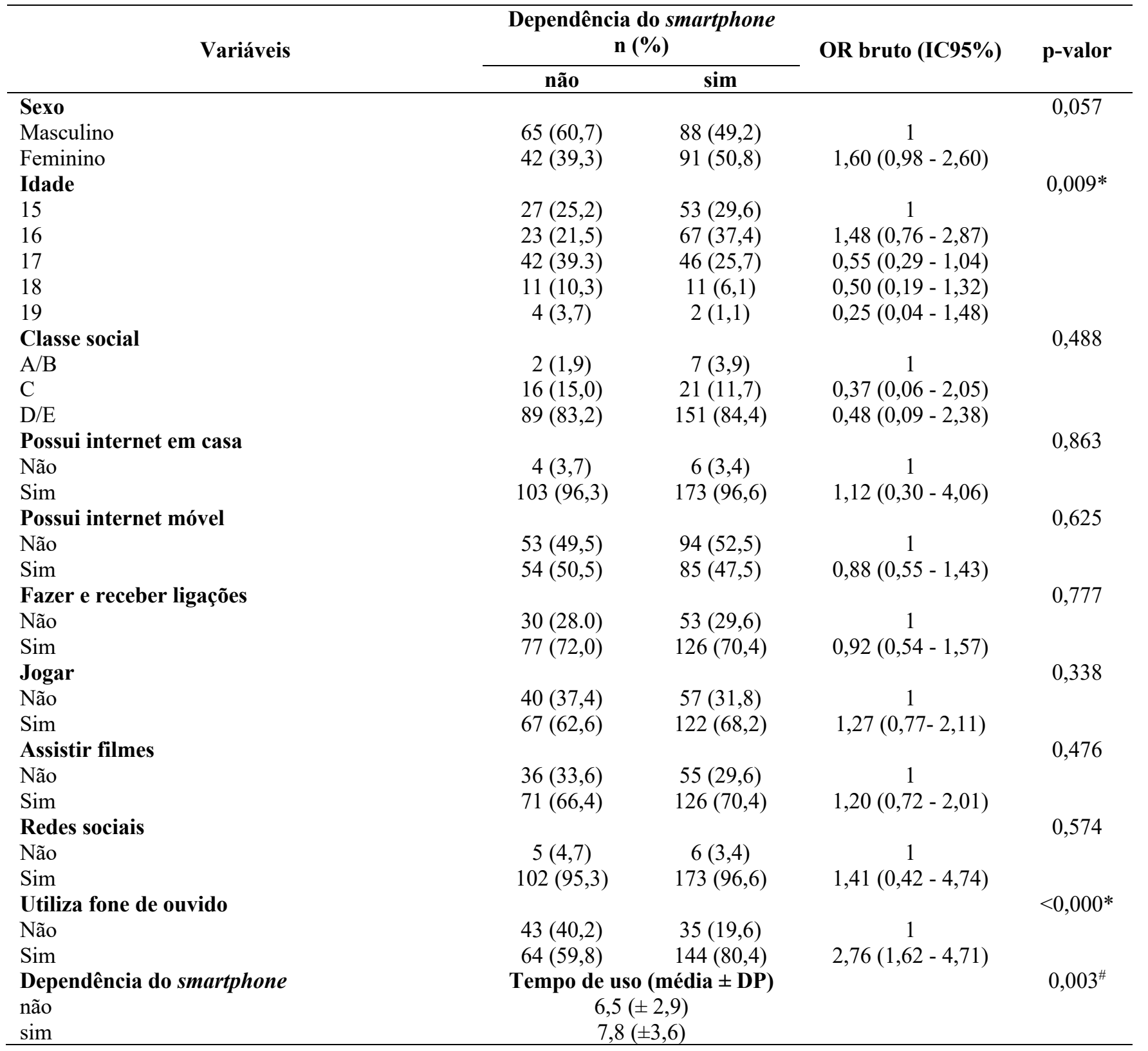

OR: odds ratio. IC95\%: intervalo de confiança de 95\%. DP: desvio padrão. *p<0,05, teste de Qui-quadrado e * teste t. Fonte: Autores.

\section{Discussão}

O presente estudo buscou conhecer o perfil de uso do smartphone pelos adolescentes e avaliar a dependência desse dispositivo, visto que de acordo com a OMS a temática abordada configura um cenário preocupante em escala mundial, referente ao uso excessivo e problemático dos dispositivos eletrônicos (WHO, 2014). Não há dúvidas de que o smartphone apresenta inúmeros benefícios, devido a facilidade de acesso à Internet e ao mesmo tempo por compactar em um único aparelho diversas opções de comunicação, praticidade e entretenimento, que fazem seus usuários permanecerem várias horas conectados (Cha \& Seo, 2018).

No tocante ao acesso à Internet, nesta pesquisa $96,5 \%$ da amostra possuía conexão em seus domicílios e 48,6\% se conectavam por dados móveis. Isto condiz com a afirmativa de que o smartphone é o principal dispositivo móvel utilizado como via de acesso à rede pelas crianças e adolescentes brasileiros (Comitê Gestor da Internet, 2019), estando presente em 
99,2\% dos domicílios do país em área urbana e 99,4\% na área rural (Instituto Brasileiro de Geografia e Estatística, 2015). Referente ao tempo de conexão por dia, o Brasil aparece em segundo lugar em um ranking mundial, com 10 horas diárias (We Are Social, 2020). Ainda em concordância com os achados, estudo realizado em 21 países emergentes constatou um aumento de 54\% no uso do celular como principal meio de acesso à Internet (Pew Research Center, 2016).

Frente a esta realidade, existem algumas lacunas presentes na literatura referente às repercussões do uso excessivo do smartphone na saúde de seus usuários (Derevensky et al., 2019). Estudos prévios alertam a necessidade de investigar o perfil de uso e a sua dependência, principalmente nos adolescentes, por serem fortemente apegados a esses dispositivos em virtude de várias razões, como jogar, estudar e relacionar-se virtualmente com outras pessoas. Isto os tornaria potencialmente dependentes (Gutiérrez et al., 2016; Elhai et al., 2017).

Em relação a estas colocações, o presente estudo constatou que as principais razões para o uso do smartphone eram acessar redes sociais, assistir filmes, jogar e, como forma de comunicação, utilizar o WhatsApp. Sobre este perfil, estudo na Coreia do Sul revelou que adolescentes acessavam as redes sociais, como Facebook (47,3\%) e Instagram (24,8\%), assistiam filmes $(17,4 \%)$ e jogavam $(20,6 \%)$ (Cha \& Seo, 2018). Em outro estudo na Suíça foi verificado que 67,3\% acessavam redes sociais, $1,8 \%$ assistiam filmes e $0,7 \%$ jogavam (Haug et al., 2015). Estes estudos mostram que os adolescentes apresentam perfil de uso semelhante devido a globalização e inexistência de barreiras no mundo digital.

No Brasil, um estudo de base nacional exibiu dados similares, mostrando que $89 \%$ das crianças e adolescentes (de 9 a 17 anos) eram usuários da Internet, destacando-se ainda que, 58\% reportaram utilizar exclusivamente o telefone celular para acessar a Internet. Os principais motivos de uso foram assistir vídeos, programas, filmes ou séries (83\%), ouvir música on-line (82\%), realizar pesquisas na Internet para trabalhos escolares (76\%) e utilizar redes sociais (68\%). Neste mesmo estudo foi mostrado que quanto mais elevada a faixa etária (15 a 17 anos) maior a intensidade das atividades online. Além disto, o WhatsApp foi a plataforma mais reportada em outro levantamento com $70 \%$, superando pela primeira vez, o Facebook (66\%) (Comitê Gestor da Internet, 2019).

No presente estudo, verificou-se que os participantes passavam mais de 7 horas/diárias usando o dispositivo e uma prevalência de dependência do smartphone de $62,6 \%$. Estudos anteriores constataram elevadas horas de uso nessa faixa etária, como na Coreia (Cha \& Seo, 2018) e no Líbano (Fares, Fares, \& Fares, 2017) com tempos superiores a 5 horas diárias. Assim como maiores percentuais de dependência foram verificados em Taiwan com 55\% (Liu, Lin, Pan, \& Lin, 2016) e Turquia com 50,6\% (Frrat et al., 2018).

As análises das subescalas do SPAI-BR, instrumento utilizado para a avaliação da dependência do smartphone, mostraram que um terço dos adolescentes apresentavam indicações de comportamento compulsivo e comprometimento funcional, e mais de $40 \%$ e de $70 \%$ apresentavam indicação de síndrome de abstinência e de tolerância, respectivamente. Em concordância, estudo libanês apontou percentuais similares para o comportamento compulsivo e comprometimento funcional, maior para a síndrome da abstinência e menor percentual para a indicação de síndrome de tolerância (Boumosleh \& Jaalouk, 2017). Em relação a estes constructos na avaliação da dependência do smartphone propostos no instrumento, é possível identificar características semelhantes ao vício da Internet e do vício em substâncias o que poderá contribuir para uma análise dos impactos biopsicossociais do uso do smartphone (Lin et al., 2014).

Ainda em relação ao SPAI-BR, constatou-se maiores proporções nos itens referente ao tempo excessivo, redução das horas de sono devido ao uso do celular e presença de desconforto e/ou dores nas costas ou olhos. De forma semelhante, estudo com adolescentes brasileiros evidenciou que 47,9\% alegam usá-lo por muito tempo e 38,7\% dizem não conseguir permanecer sem seus smartphones (Correr \& Faidiga, 2017). Outro inquérito realizado no Sul de Taiwan com escolares verificou que quanto maior o grau de dependência, menor é a qualidade do sono, e que $90 \%$ de sua amostra utiliza o celular antes de dormir. Neste estudo, os autores constataram que quanto mais tempo gasto no dispositivo maior prejuízo na qualidade do sono (Wang, 
Chen, Yang, \& Lin, 2019). Em relação ao desconforto musculoesquelético, outros estudos também atestaram queixa de dores na região das costas e nos olhos (Queiroz, Lourenço, Silva, Lourenço, \& Silva, 2018), e a ocorrência de sintomas oculares como tensão ocular (12\%), olhos secos (7\%) e miopia (3\%) (Fares et al., 2017).

Nos últimos anos, o uso do celular vem sendo frequentemente associado a fatores potencialmente prejudiciais à saúde e em vários aspectos da vida diária. Contudo, ainda há necessidade de investigar os mecanismos comportamentais e neurobiológicos envolvidos no uso problemático do smartphone, acompanhando as evidências descritas em outros comportamentos viciantes. Um modelo teórico foi proposto para orientar e apoiar estudos sobre esta temática, que envolviam três vias possíveis com características heterogênea e multifacetada, que seriam "via de reafirmação excessiva", "via impulsiva antissocial" e "via de extroversão" (Billieux, Maurage, Lopez-Fernandez, Kuss, \& Griffiths, 2015). Em paralelo, no presente estudo algumas dessas características que estão neste modelo foram identificadas em elevado percentual, como a dependência do smartphone e tempo de uso excessivo, sendo redes sociais, jogos online e WhatsApp os principais aplicativos.

Por fim, algumas recomendações são propostas com base nos achados deste estudo como, a implantação de campanhas educativas e orientações sobre a temática em diferentes cenários, com foco na promoção do uso consciente do smartphone pelos adolescentes, em consonância com a Sociedade Brasileira de Pediatria (2016) no que se refere ao uso de dispositivos eletrônicos.

São reconhecidas algumas limitações no presente estudo, como a ausência de questionários específicos que avaliem o tempo de uso e a finalidade de uso desse dispositivo. Em relação a idade e outras variáveis socioeconômicas da amostra, estas foram limitadas em virtude do local da pesquisa, o que dificulta a generalização dos dados. No entanto, apesar destas limitações, os achados dessa pesquisa podem contribuir para o fomento e discussão da temática estudada.

\section{Conclusão}

Dentre as razões para o uso do smartphone pelos adolescentes destacam-se acessar as redes sociais, assistir filmes e jogar, bem como usar o WhatsApp para fins de comunicação. Evidenciou-se uma elevada prevalência de dependência do smartphone e tempo de uso excessivo pelos adolescentes, mostrando uma coexistência entre essas variáveis. Diante disto, alerta-se para a necessidade de campanhas educativas e orientações divulgadas, principalmente pelas redes sociais e aplicativos comumente usados por essa população para o uso consciente do smartphone, prevenção e redução de agravos à saúde física $\mathrm{e}$ mental. Ademais, encoraja-se a realização de estudos longitudinais para aprofundar as questões relacionadas ao impacto desta problemática no âmbito da saúde pública.

\section{Agradecimentos}

A Secretaria da Educação do Estado do Ceará (Seduc) e as Escolas Estaduais de Educação Profissional (EEEP).

\section{Referências}

Billieux, J., Maurage, P., Lopez-Fernandez, O., Kuss, D. J., \& Griffiths, M. D. (2015). Can Disordered Mobile Phone Use Be Considered a Behavioral Addiction? An Update on Current Evidence and a Comprehensive Model for Future Research. Current Addiction Reports, 2(2), $156-162$.

Bloch, K. V., \& Klein, C. H. (2009). Estudos Seccionais. In: Medronho, R. A. Epidemiologia. Saraiva.

Boumosleh, J. M., \& Jaalouk, D. (2017). Depression, anxiety and smartphone addiction. PLoS ONE, 12(8), 1-14.

Comitê Gestor da Internet. (2019). Pesquisa sobre o uso da internet por crianças e adolescentes no Brasil: TIC kids online Brasil 2018 / Survey on internet use by children in Brazil: ICT kids online Brazil 2018.

Cha, S. S., \& Seo, B. K. (2018). Smartphone use and smartphone addiction in middle school students in Korea: Prevalence, social networking service, and game use. Health Psychology Open, 5(1). 
Correr, R., \& Faidiga, M. T. B. (2017). O uso do celular por adolescentes: Impactos nos relacionamentos. Adolescencia e Saude, 14(2), 24-39.

Derevensky, J. L., Hayman, V., \& Lynette Gilbeau. (2019). Behavioral Addictions: Excessive Gambling, Gaming, Internet, and Smartphone Use Among Children and Adolescents. Pediatric Clinics of North America, 66(6), 1163-1182.

Elhai, J. D., Dvorak, R. D., Levine, J. C., \& Hall, B. J. (2017). Problematic smartphone use: A conceptual overview and systematic review of relations with anxiety and depression psychopathology. Journal of Affective Disorders, 207, 251-259.

Fares, J., Fares, M. Y., \& Fares, Y. (2017). Musculoskeletal neck pain in children and adolescents: Risk factors. Surgical Neurology International, 8(72), 1-5.

Fırat, S., Gül, H., Sertçelik, M., Gül, A., Gürel, Y., \& Kılıç, B. G. (2018). The relationship between problematic smartphone use and psychiatric symptoms among adolescents who applied to psychiatry clinics. Psychiatry Research, 270, 97-103.

Gutiérrez, J. D. S., de Fonseca, F. R., \& Rubio, G. (2016). Cell-phone addiction: A review. Frontiers in Psychiatry, 7(175), 1-15.

Haug, S., Paz Castro, R., Kwon, M., Filler, A., Kowatsch, T., \& Schaub, M. P. (2015). Smartphone use and smartphone addiction among young people in Switzerland. Journal of Behavioral Addictions, 4(4), 299-307.

Instituto Brasilerio de Estatística e Geografia. (2018). Uso de internet, televisão e celular no Brasil. Instituto Brasileiro de Geografia e Estatística, 2017, https://educa.ibge.gov.br/jovens/materias-especiai.

Instituto Brasileiro de Geografia e Estatística. IBGE. (2015). Pesquisa Nacional por Amostra de Domicílios. Acesso à internet e à televisão e posse de telefone móvel celular para uso pessoal : 2015. (IBGE). https://biblioteca.ibge.gov.br/visualizacao/livros/liv99054.pdf

Jiang, Z., \& Zhao, X. (2016). Self-control and problematic mobile phone use in Chinese college students: The mediating role of mobile phone use patterns. BMC Psychiatry, 16(1), 1-8.

Khoury, J. M., De Freitas, A. A. C., Roque, M. A. V., Albuquerque, M. R., Das Neves, M. D. C. L., \& Garcia, F. D. (2017). Assessment of the accuracy of a new tool for the screening of smartphone addiction. PLOS ONE, 12(5), 1-13.

Lee, H., Kim, J. W., \& Choi, T. Y. (2017). Risk factors for smartphone addiction in Korean adolescents: Smartphone use patterns. Journal of Korean Medical Science, 32(10), 1674-1679.

Lin, Y. H., Chang, L. R., Lee, Y. H., Tseng, H. W., Kuo, T. B. J., \& Chen, S. H. (2014). Development and validation of the Smartphone Addiction Inventory (SPAI). PLoS ONE, 9(6), e98312. https://doi.org/10.1371/journal.pone.0098312

Liu, C. H., Lin, S. H., Pan, Y. C., \& Lin, Y. H. (2016). Smartphone gaming and frequent use pattern associated with smartphone addiction. Medicine (United States), 95(28), 1-4.

Pew Research Center. (2016). Smartphone Ownership and Internet Usage Continues to Climb in Emerging Economies. Pew Research Center, pp. 1-5.

Queiroz, L. B., Lourenço, B., Silva, L. E. V., Lourenço, D. M. R., \& Silva, C. A. (2018). Musculoskeletal pain and musculoskeletal syndromes in adolescents are related to electronic devices. Jornal de Pediatria (Versão Em Português), 94(6), 673-679.

Schweizer, A., Berchtold, A., Barrense-Dias, Y., Akre, C., \& Suris, J. C. (2017). Adolescents with a smartphone sleep less than their peers. European Journal of Pediatrics, 176(1), 131-136.

Secretaria da Educação do Estado do Ceará. (2019). Ensino Médio e Profissional. Retrieved from https://www.seduc.ce.gov.br/escolas-estaduais-de-educacaoprofissional-no-ceara/

Sociedade Brasileira de Pediatria. (2016). Saúde de Crianças e Adolescentes na Era Digital. https://www.sbp.com.br/fileadmin/user_upload/2016/11/19166dMOrient-Saude-Crian-e-Adolesc.pdf

Wang, P. Y., Chen, K. L., Yang, S. Y., \& Lin, P. H. (2019). Relationship of sleep quality, smartphone dependence, and health-related behaviors in female junior college students. PLOS ONE, 14(4), 1-12.

We Are Social. (2021). Digital 2021 - We Are Social. https://wearesocial.com/digital-2021

World Health Organization. (2014). Public Health Implications of Excessive Use of the Internet, Computers, Smartphones and Similar Electronic Devices Meeting report. http://www.ncbi.nlm.nih.gov/pubmed/21661372

Xie, Y., Szeto, G., \& Dai, J. (2017). Prevalence and risk factors associated with musculoskeletal complaints among users of mobile handheld devices: A systematic review. Applied Ergonomics, 59, 132-142. 